\title{
Managing parallel COVID-19 epidemics in a single country
}

\author{
Samuel SY Wang ${ }^{1}$ *, BMed, MD, Winnie ZY Te0 ${ }^{1,2}$, MB BcH, BAO, LY Hsü3, MB, BS, MPH (Harvard) \\ ${ }^{1}$ Fast Program, Alexandra Hospital, National University Hospital System, Singapore \\ ${ }^{2}$ Department of Haematology-Oncology, National University Cancer Institute Singapore (NCIS), National University Health System, \\ Singapore \\ ${ }^{3}$ NUS Saw Swee Hock School of Public Health (Primary), Singapore \\ *Corresponding author: samuel.wang@mohh.com.sg \\ Hong Kong Med J 2021;27:145-7 \\ https://doi.org/10.12809/hkmj209083
}

This article was published on 9 Apr 2021 at www.hkmj.org.
Tackling the coronavirus disease 2019 (COVID-19) pandemic involves breaking the chain of infection through social distancing, testing and quarantine in an attempt to not overwhelm the health services, and developing effective vaccines. ${ }^{1}$ Mask wearing is also an integral part of controlling the spread of COVID-19 through a combination of source control and personal protection for the mask wearer. ${ }^{2}$ Before the availability of effective vaccines, testing, quarantine and social distancing are paramount in stabilising infection rates and protecting healthcare systems from being overwhelmed. For countries with stable infection rates the focus is reopening their economies and resumption of normal clinical services whilst being vigilant for subsequent pandemic waves.

In Singapore, COVID-19 infections decreased after a high in April 2020, raising confidence that the economy would reopen. ${ }^{3}$ What is interesting about the COVID-19 pandemic in Singapore is its dual nature, because COVID-19 cases in Singapore are divided into foreign dormitory worker (FW) cases and community cases. ${ }^{3}$ Singapore has a population of 5.6 million people with a total foreign workforce of 1427500 of which 261900 are FW staying in 42 FW dormitories; 180000 of them work in the construction industry. In May 2020, community cases were under control with minimal imported cases and fewer than 10 daily cases. In contrast, the bulk of the daily infections were FW cases, which decreased from the high in April but remained in the high double-digit range after the completion of testing in the majority of FW dormitories. Singapore was in a unique situation of simultaneously managing two very different COVID-19 outbreaks: one in the general community and another among FWs. This proved quite challenging when deciding when and how to reopen the economy whilst remaining vigilant about future outbreaks.

The factors responsible for the more severe outbreak among FWs can be attributed to them being a marginalised and economically vulnerable population living in overcrowded and less sanitary accommodations. This compromises social distancing effectiveness and facilitates severe acute respiratory syndrome coronavirus 2 (SARS-CoV-2) infections. Construction sites where safe distancing practices were difficult to enforce and FWs from various dormitories mixed, drive the outbreak among FWs. ${ }^{4}$ Existing poor health literacy, low education levels, and cultural and language barriers impeded communication of public healthcare policies. In some cases, FWs underreported symptoms or avoided medical help, fearing loss of employment or income. These basic challenges, including language or cultural barriers, healthcare communication, and overcrowded or unsanitary living conditions, are similar to the challenges observed in outbreaks in developing countries. ${ }^{5-7}$

A multi-pronged approach was employed to tackle the pandemic among FWs. Those working in essential services were first separated and relocated to new self-isolation facilities away from the dormitories viewed as sources of infection. ${ }^{8}$ To house the large number of infected patients while providing rudimentary but relatively effective monitoring and healthcare, community care facilities were rapidly developed using existing exhibition centres to more than double Singapore's hospital bed capacity.

Next an aggressive campaign of testing symptomatic and asymptomatic FWs was undertaken. ${ }^{8}$ Those FWs who were older, had comorbidities, and were symptomatic for severe COVID-19 as characterised by dyspnoea and worsening fevers were then brought to acute hospitals for observation and risk stratifying using chest radiographs and blood tests. ${ }^{8}$ Once observed to be clinically improving, they were transferred to community care facilities for further observation until deemed to be non-infective either by swab tests or by duration of illness. ${ }^{8}$

However, an unintended but concurrent strategy to testing and isolating was the natural acquisition of herd immunity among FWs. Fortunately, despite the high infection rates among FWs the mortality and morbidity rates remained low. The majority of FWs had mild symptoms and 
uncomplicated recovery, likely owing to the patient demographics: FWs are primarily young or middleaged males with minimal chronic medical comorbidities and working in physically demanding jobs. Thus, despite the controversy, in the right population group with low mortality and morbidity despite high infection rates, inadvertent natural acquisition of herd immunity might assist in controlling the outbreak. ${ }^{9}$ Immunity to COVID-19 through natural infection is likely to be dependent upon both cell-dependent immunity from SARS-CoV-2-specific CD4+ and CD8+ memory T cells and humoral immunity from anti-SARS-CoV-2 immunoglobulin $\mathrm{G}$ and immunoglobulin $\mathrm{A}$ though further research is needed to better understand it. ${ }^{10}$

Even with a reduction in the number of COVID-19 cases among FWs, continued social distancing and isolation among FWs to break the chain of infection remained important, to prevent transmission into the wider community. ${ }^{8}$ However, this social distancing and isolation took a toll on FWs already viewed as a voiceless subset of the Singaporean population. Despite having their salaries heavily subsidised by the Singapore government, FWs were confined for months without appeal under conditions of tremendous uncertainty. This led to increased mental health issues and even suicides. Despite the controversy, social distancing between the community and FWs was critical and remains an important part of the public healthcare policy as Singapore restarts its economy.

Measures were also undertaken to control the outbreak in the community. First was minimisation of economic activity through shutting down nonessential industries and services which were unable to digitise. ${ }^{11}$ This enabled the general population to effectively practise social distancing. Building upon this was the development of contact tracing applications-including the "TraceTogether" and "SafeEntry" national digital check-in system-and encouraging their uptake among the population. ${ }^{11}$ These measures facilitated contact tracing of individuals exposed to a confirmed positive COVID-19 case. $^{11}$ Next was increasing capacity to enable aggressive testing for SARS-CoV-2 in asymptomatic or symptomatic individuals in acute hospitals, aged care facilities, or in general practitioner clinics. ${ }^{11}$ These data enabled healthcare planners to identify infection patterns and clusters, and to enact proactive measures to control them. ${ }^{11}$

For TWs and in the community, discharge from isolation facilities required a double-negative COVID-19 polymerise chain reaction test from a nasopharyngeal swab. As an extra precaution, discharged FWs had to stay in clean dormitories, whereas community cases were discharged home. The rationale was the great difference in the infection rates between community and FW populations at that time.

Based on the Singaporean experience in managing COVID-19, it may be prudent to be more proactive to control disease outbreaks. Future robust investment into public healthcare infrastructure which enables a rapid upscaling of testing and isolation facilities might be a feature of future healthcare planning. Additionally, the pandemic in Singapore has also highlighted the importance of caring for the health of marginalised communities in Singapore. In a small and densely populated city, public health is as strong as its weakest link and is therefore everyone's responsibility. Specific policies have been formulated to minimise overcrowding and ensure improved sanitation in FW dormitories. Furthermore, employers will be expected to ensure better healthcare accessibility for FWs. This may raise the cost of business but after experiencing the COVID-19 worldwide pandemic, higher sanitation and hygiene may be accepted as a new normal.

\section{Author contributions}

Concept or design: SSY Wang.

Acquisition of data: SSY Wang.

Analysis or interpretation of data: All authors.

Drafting of the manuscript: SSY Wang.

Critical revision of the manuscript for important intellectual content: All authors.

All authors had full access to the data, contributed to the study, approved the final version for publication, and take responsibility for its accuracy and integrity.

\section{Conflicts of interest}

All authors have disclosed no conflicts of interest.

\section{Funding/support}

This commentary received no specific grant from any funding agency in the public, commercial, or not-for-profit sectors.

\section{References}

1. Anderson RM, Heesterbeek H, Klinkenberg D, Hollingsworth TD. How will country-based mitigation measures influence the course of the COVID-19 epidemic? Lancet 2020;395:931-4.

2. Schünemann HJ, Akl EA, Chou R, et al. Use of facemasks during the COVID-19 pandemic. Lancet Respir Med 2020;8:954-5.

3. Ministry of Health Singapore. COVID-19 situation report. Available from: https://covidsitrep.moh.gov.sg/. Accessed 5 Apr 2021.

4. Lan FY, Wei CF, Hsu YT, Christiani DC, Kales SN. Workrelated COVID-19 transmission in six Asian countries/ areas: A follow-up study. PLoS One 2020;15:e233588.

5. Saraya A. Indian response to COVID-19: Expertise and transparency. Indian $J$ Public Health 2020;64(Supplement):S243-4.

6. Chetterje P. Gaps in India's preparedness for COVID-19 control. Lancet Infect Dis 2020;20:544.

7. Marson FA, Ortega MM.COVID-19 in Brazil. Pulmonology 
2020;26:241-4.

8. Chew MH, Koh FH, Wu JT, et al. Clinical assessment of COVID-19 outbreak among migrant workers residing in a large dormitory in Singapore. J Hosp Infect 2020;106:2023.

9. Kwok KO, Lai F, Wei WI, Wong SY, Tang JW. Herd immunity-estimating the level required to halt the
COVID-19 epidemics in affected countries. J Infect 2020;80:e32-3.

10. Stephens DS, McElrath MJ. COVID-19 and the path to immunity. JAMA 2020;324:1279-81.

11. Lee VJ, Chiew CJ, Khong WX. Interrupting transmission of COVID-19: lessons from containment efforts in Singapore. J Travel Med 2020;27:taaa039. 